\title{
Phylogeny of Hirsutella species (Ophiocordycipitaceae) from the USA: remedying the paucity of Hirsutella sequence data
}

\author{
D. Rabern Simmons ${ }^{1 *}$, Ryan M. Kepler ${ }^{2}$, Stephen A. Rehner ${ }^{2}$, and Eleanor Groden ${ }^{3}$
}

${ }^{1}$ University of Florida, Institute of Food and Agricultural Sciences, School of Forest Resources and Conservation, PO Box 110410, Gainesville, FL 32611-0410, USA; corresponding author e-mail: rabernsimmons@ufl.edu

2USDA Agricultural Research Service, Systematic Mycology and Microbiology Laboratory, Beltsville, MD 20705, USA

${ }^{3}$ University of Maine, School of Biology \& Ecology, 5722 Deering Hall, Orono, ME 04473-5722, USA

\begin{abstract}
Hirsutella (Ophiocordycipitaceae: Hypocreales) is a genus of insect, mite, and nematode pathogens with an asexual morph, which generally produce a mucilaginous cluster of one or several conidia on phialides that are basally subulate and taper to a fine neck. The generic name Hirsutella has been proposed for suppression in favour of Ophiocordyceps as a consequence of the ending of dual nomenclature for different morphs of pleomorphic fungi in 2011. Though the generic name is well established, geographically dispersed, and speciose, exceptionally few sequences are available in online databases. We examined 46 isolates of 23 Hirsutella species from the USA, curated by the USDA-ARS Collection of Entomopathogenic Fungal Cultures (ARSEF Culture Collection), that previously had not been molecularly characterized and produced a phylogeny of these organisms; we included previously published Hirsutella and Ophiocordyceps taxa. In producing the largest phylogeny of Hirsutella isolates so far, we provide: (1) context for discussing previously-hypothesized relationships; (2) evidence for revisions as taxonomic transitions move forward; and (3) available molecular data to be incorporated into further evolutionary studies of Ophiocordycipitaceae.
\end{abstract}

Key words:

18S rDNA

biocontrol

entomopathogenic fungi

Hypocreales

pleomorphic fungi

$r p b 1$

tef1

Article info: Submitted: 3 July 2015; Accepted: 28 September 2015; Published: 26 October 2015.

\section{INTRODUCTION}

Hirsutella (Patouillard 1892) contains the names of over 70 species of asexually-reproducing pathogens of insects, mites, and nematodes that are distributed mainly within Ophiocordycipitaceae (Kepler et al. 2013, Quandt et al. 2014), though the genus is usually considered to be associated with the genus Ophiocordyceps typified by a sexual morph (Sung et al. 2007). Hirsutella species are distinguished from other asexually typified genera by the basally swollen or subulate phialides that taper to an apex, where a mucilaginous packet of one or several conidia forms. Since being described, the morphological concept of the genus has expanded to include species with traits either varying or lacking from a general suite of characters (e.g. phialides without basal inflation, conidia unbounded by a mucilaginous sheath, polyphialidic conidiogenous cells), thereby making morphological inferences of interspecific relationships confusing, if not impossible.

Hirsutella is one of the many fungal taxa typified by asexual morphs affected by the ending of dual nomenclature for pleomorphic fungi in 2011 (McNeill et al. 2012). Quandt et al. (2014) argued that Hirsutella should be suppressed in favour of Ophiocordyceps because of a lack of type material for clarification and the larger task of new combinations to be made if Hirsutella were selected. Also, the desire to preserve the term 'cordyceps' within the genus to reflect the cultural and economic importance of O. sinensis (Sung et al. 2007) was expressed. Indeed, the adoption of Ophiocordyceps for a new species only known from a Hirsutella morph already has been put to practice. Simmons et al. (2015) described Ophiocordyceps myrmicarum from asexually reproducing "Hirsutella" cultures, isolated from European fire ant (Myrmica rubra) populations in Maine, for which the sexual morph was not observed. The suppression of the generic name, however, is not to imply a lack of knowledge or importance of these fungi, but instead should act to propel research into the phylogeny of these organisms so that they may be incorporated into the larger evolutionary history and impending taxonomic revisions of the family Ophiocordycipitaceae.

Hodge (1998) produced the only monograph of Hirsutella, and was among the first to produce a molecular phylogeny of this genus to determine interspecific relationships that could not be deduced through morphological comparisons. Hodge examined 13 isolates and determined that Hirsutella was monophyletic with three Harposporium isolates under parsimony analyses of nuclear ITS rDNA sequences. Hodge (1998: 210) stated, however, that ". . . analyses that include more data from different areas of the genome and more taxon sampling are in order before nomenclatural changes can be suggested." Indeed, Chaverri et al. (2005) described Podocrella peltata with supporting data from the 28S rDNA region from a Harposporium sp. (ARSEF 5410), which Hodge (1998) had found belonged within the Hirsutella monophyletic

\section{๑) 2015 International Mycological Association}

You are free to share - to copy, distribute and transmit the work, under the following conditions:

Attribution: $\quad$ You must attribute the work in the manner specified by the author or licensor (but not in any way that suggests that they endorse you or your use of the work).

Non-commercial: $\quad$ You may not use this work for commercial purposes.

No derivative works: You may not alter, transform, or build upon this work.

For any reuse or distribution, you must make clear to others the license terms of this work, which can be found at http://creativecommons.org/licenses/by-nc-nd/3.0/legalcode. Any of the above conditions can be waived if you get permission from the copyright holder. Nothing in this license impairs or restricts the author's moral rights. 
group. Quandt et al. (2014) concurred with the synonymy of Harposporium and Podocrella, but placed these taxa in a clade disparate from Ophiocordyceps, and thus Hirsutella s. str.; the authors also argued for the suppression of the generic name Podocrella in favour of Harposporium, citing the nomenclatural precedence of Harposporium (i.e. priority of publication), and the shared conidia morphology within the clade.

Since the study of Hodge (1998), little phylogenetic work has been conducted with Hirsutella, except for limited discussion in descriptions of new species (Seifert \& Boulay 2004, Evans et al. 2010, Ciancio et al. 2013, Simmons et al. 2015). The lack of available molecular data, however, has made the task of species designation difficult and potentially misleading, in that by limiting the taxa to which an investigator compares a new species risks falsely inflating the genus with a species that has already been described, but for which molecular data have not been generated.

To populate online databases with sequence data from a well-curated and accessible fungal collection, we examined isolates named as belonging to Hirsutella in the USDA-ARS Collection of Entomopathogenic Fungal Cultures (ARSEF Culture Collection). We produced a phylogeny of these taxa based on three genetic loci, and we assembled morphological data of these species, or specific isolates from the literature. We discuss the relationships of these species hypothesized in Hodge's monograph and other studies, provide evidence for revisions as taxonomic transitions move forward under the new rules (McNeill et al. 2012), and make available molecular data to facilitate the use of these fungi and their associated data in further evolutionary studies of Hirsutella, Ophiocordyceps, and Ophiocordycipitaceae.

\section{MATERIALS AND METHODS}

\section{DNA extraction, PCR amplification and sequencing}

Cultures of ARSEF isolates were provided by Richard A. Humber (ARSEF Culture Collection, Ithaca, NY), and tissues were lyophilized at the USDA-ARS Systematic Mycology and Microbiology Laboratory (Beltsville, MD), before being sent to the University of Maine (Orono, ME) for DNA extraction. Genomic DNA was extracted from 47 ARSEF Hirsutella isolates (Table 1) with $\mathrm{MO}$ BIO UltraClean ${ }^{\circledR}$ Microbial DNA isolation kits (MO BIO Laboratories, Carlsbad, CA) following the manufacturer's protocol, with further disruption of the tissue with a Mini-BeadBeater 16 (Biospec Products, Bartlesville, OK) for $3 \mathrm{~min}$. Stock extracts were diluted before amplifications to decrease inhibitory contaminants from the extraction kit spin filters.

Amplifications were conducted in Eppendorf Mastercyler ${ }^{\circledR}$ gradient (Eppendorf Nother America, Westbury, NY) or BioRad T100 (Bio-Rad Laboratories, Hercules, CA) thermal cyclers. Final concentrations of PCR reagent solutions in $25 \mu \mathrm{L}$ were: (1) $1 \times$ Promega GoTaq PCR buffer (Promega, Madison, WI); (2) $1.5 \mathrm{mM} \mathrm{MgCl}$; (3) $0.2 \mathrm{mM}$ dNTPs; (4) 0.5 $\mu \mathrm{M}$ of each primer; (5) $1 \mathrm{U}$ GoTaq DNA polymerase; and (6) 0.02-0.2 ng extracted DNA. PCR conditions for tef1, rpb1, $18 \mathrm{~S}$ rDNA, and 28S rDNA were as by Johnson et al. (2009) and for nuclear ITS rDNA as by Simmons et al. (2015).
Primer sequences were obtained from previous works or designed in Geneious (Geneious version 7.1.8). Primer combinations used for amplifications were: (1) $983 \mathrm{~F}$ and 2218R (Carbone \& Kohn 1999, Rehner \& Buckley 2005) for tef1; (2) RPB1-313F (5'-YTGGARATTGTCTGCCAYAAY-3') and RPB1-1149R (5'-RCGCTTDCCCATCAAGTTGC-3') or RPB1CrW (5'-CCNGCDATNTCRTTRTCCATRWA-3'), which was derived from RPB1Cr (Castlebury et al. 2004), for rpb1; (3) NS1 and NS4 (White et al. 1990) for 18S rDNA; (4) LROR and LR5 (Vilgalys \& Hester 1990, Rehner \& Samuels 1994) for 28S rDNA; and (5) ITS1F or ITS5 and ITS4 (White et al. 1990, Gardes \& Bruns 1993) for nuclear ITS rDNA. Amplified products were visualized and gel extracted as by Simmons (2011) and sequenced by the University of Maine Sequencing Facility. All products were sequenced with the same primers by which they were amplified, except some tef1 sequences, which were additionally sequenced with primers $1577 \mathrm{~F}$ and 1567R (Rehner \& Buckley 2005).

\section{Phylogenetic analyses}

Chromatograms were manually inspected for ambiguous nucleotides, assembled, and aligned with tef1, rpb1, and 18S rDNAsequences of Ophiocordycipitaceae (Table 1) in Geneious (Geneious version 7.1.8). PartitionFinder v1.1.1 (Lanfear et al. 2012) recommended that the super-matrix of the three loci alignments be divided into seven partitions for phylogenetic consideration: one partition for the 18S rDNA alignment and for each of the three codon positions in both protein encoding genes tef1 and rpb1. The Akaike information criterion (AIC) in jModeltest 0.1.1 (Guindon \& Gascuel 2003, Posada 2008) was used to select the nucleotide substitution model for each partition. Maximum likelihood (ML) phylogenetic analyses were conducted in GARLI 2.01 (Zwickl 2006) with the recommended partition parameters to determine the best tree topology (Fig. 1) and bootstrap support values from 500 search replicates, which were summarized in SumTrees (Sukumaran \& Holder 2010). Bayesian posterior probabilities (BPP) were estimated with the same partition parameters in an analysis conducted in MrBayes 3.1.2 (Ronquist \& Huelsenbeck 2003), in which two runs of four chains each were executed simultaneously for 5000000 generations, with sampling every 500 generations. SumTrees was used to compute BPP from a summary of 7501 trees retained after a burn-in of the first 2500 trees collected.

\section{RESULTS}

The super-matrix phylogenetic analyses included tef1, rpb1, and $18 S$ rDNA sequences from 47 ARSEF isolates referred to Hirsutella, 46 of which were represented by novel sequence data from these genetic loci (Table 1). One isolate, Hirsutella gigantea (ARSEF 30), had been investigated previously for a phylogeny with tef1 sequence data (Simmons et al. 2015), but additional data for $r p b 1$ from this culture are provided in the present study. We excluded alignments of nuclear ITS and $28 \mathrm{~S}$ rDNA regions for combined phylogenetic analyses, because they generated incongruent tree topologies, but we report these sequences for further molecular identification of ARSEF cultures. The super-matrix of tef1, rpb1, and $18 \mathrm{~S}$ rDNA was deposited in TreeBASE submission 17863. 


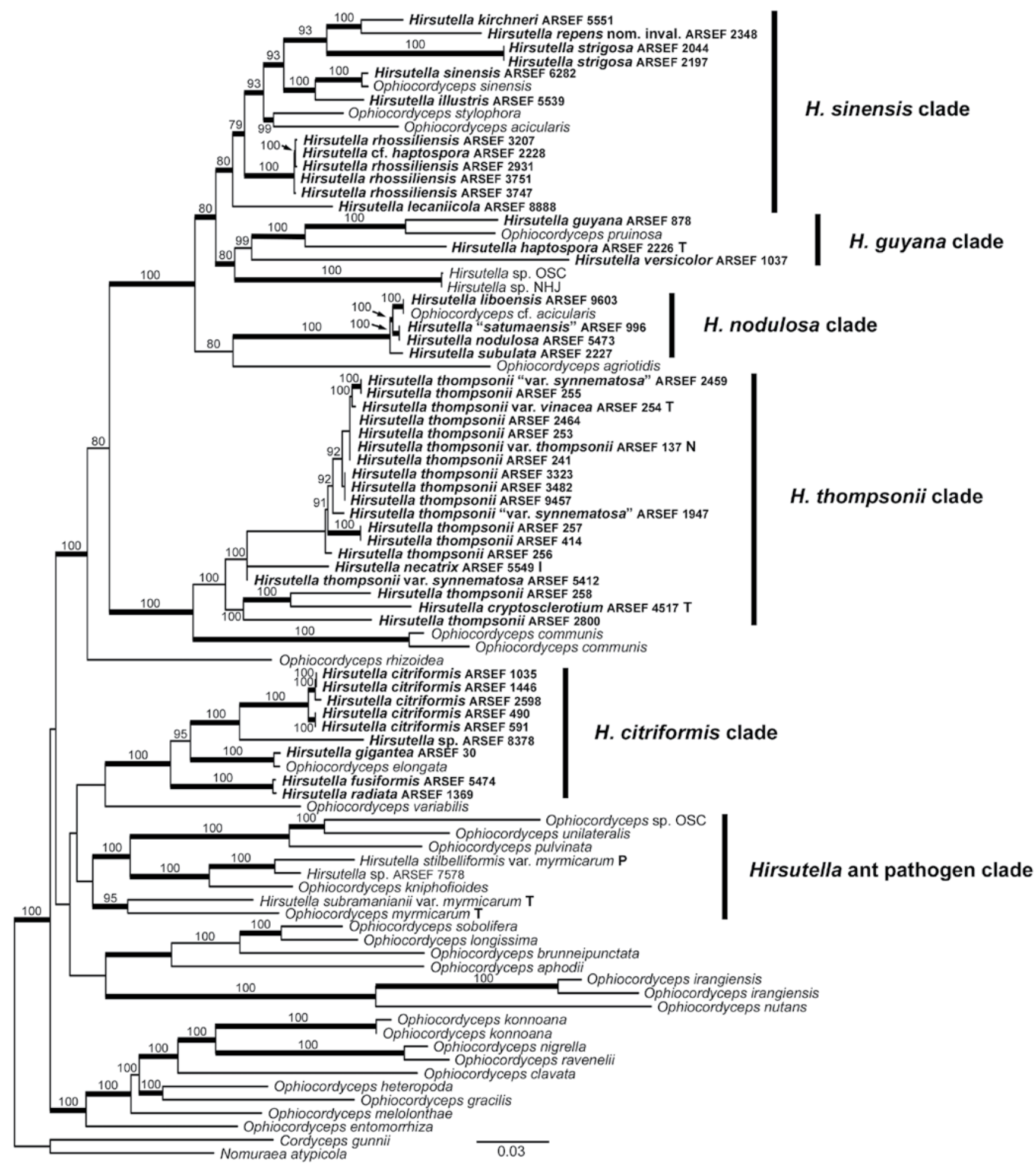

Fig. 1. Best ML tree from GARLI analysis of combined tef1, rpb1, and 18S rDNA datasets, with taxa represented by sequences obtained in this study in bold. Culture designations of Hirsutella species and Ophiocordyceps myrmicarum indicated as: I, ex-isotype; N, ex-neotype; P, ex-paratype; T, ex-type. Values at nodes represent ML bootstrap percentages $\geq 70 \%$ from a summary of 500 replicates, and branches in bold represent BPP $\geq 95 \%$.

The best ML tree from GARLI analysis (Fig. 1) grouped Hirsutella and Ophiocordyceps taxa monophyletic to Purpureocillium (Nomuraea atypicola) and Drechmeria (Cordyceps gunnii) outgroup taxa, which are also classified within Ophiocordycipitaceae (Quandt et al. 2014). We recognize six distinct groups to facilitate discussion below, though support values for these groups vary. These six groups include $H$. citriformis, $H$. thompsonii, $H$. nodulosa, $H$. guyana, $H$. sinensis, and the Hirsutella ant pathogen clade. 


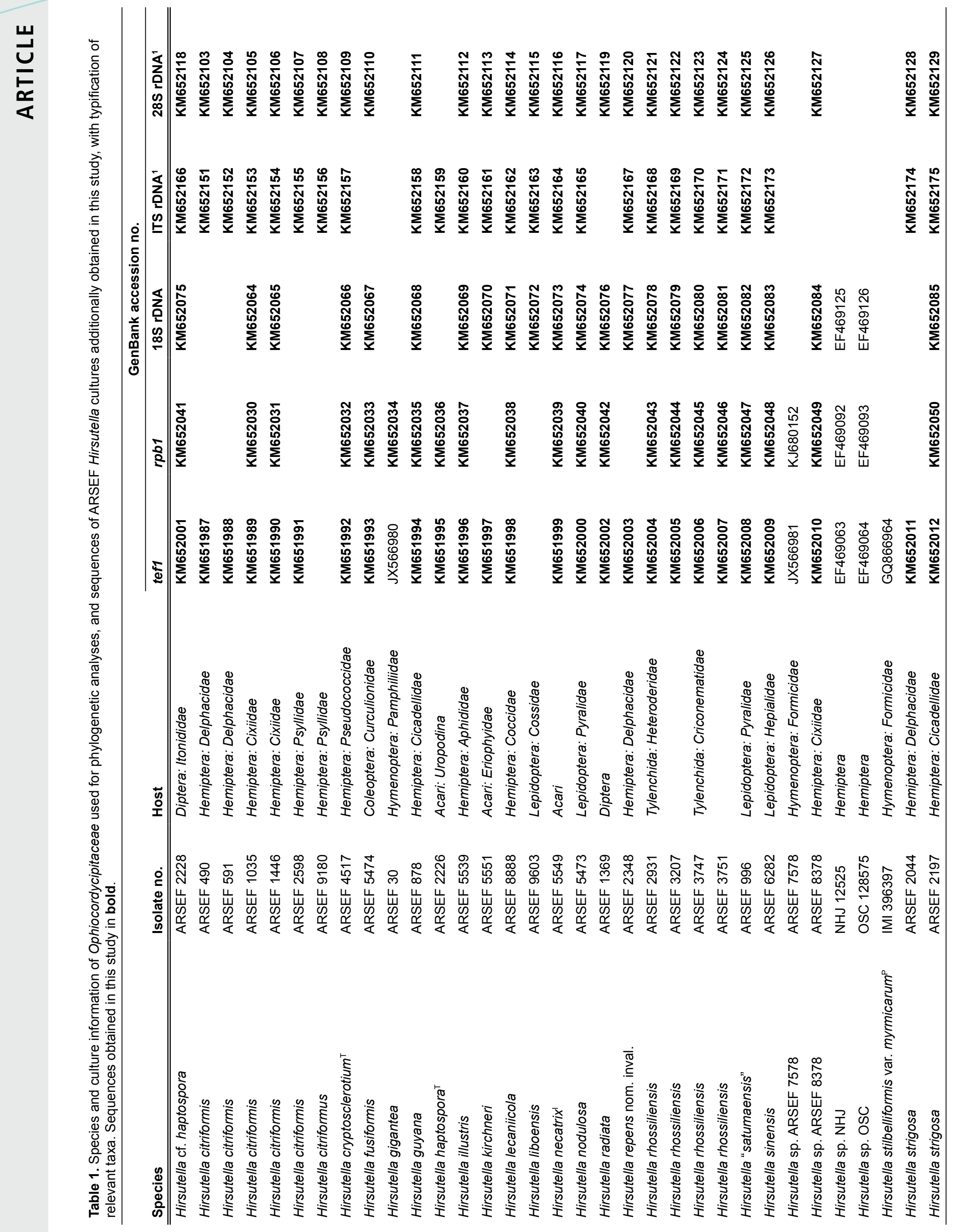




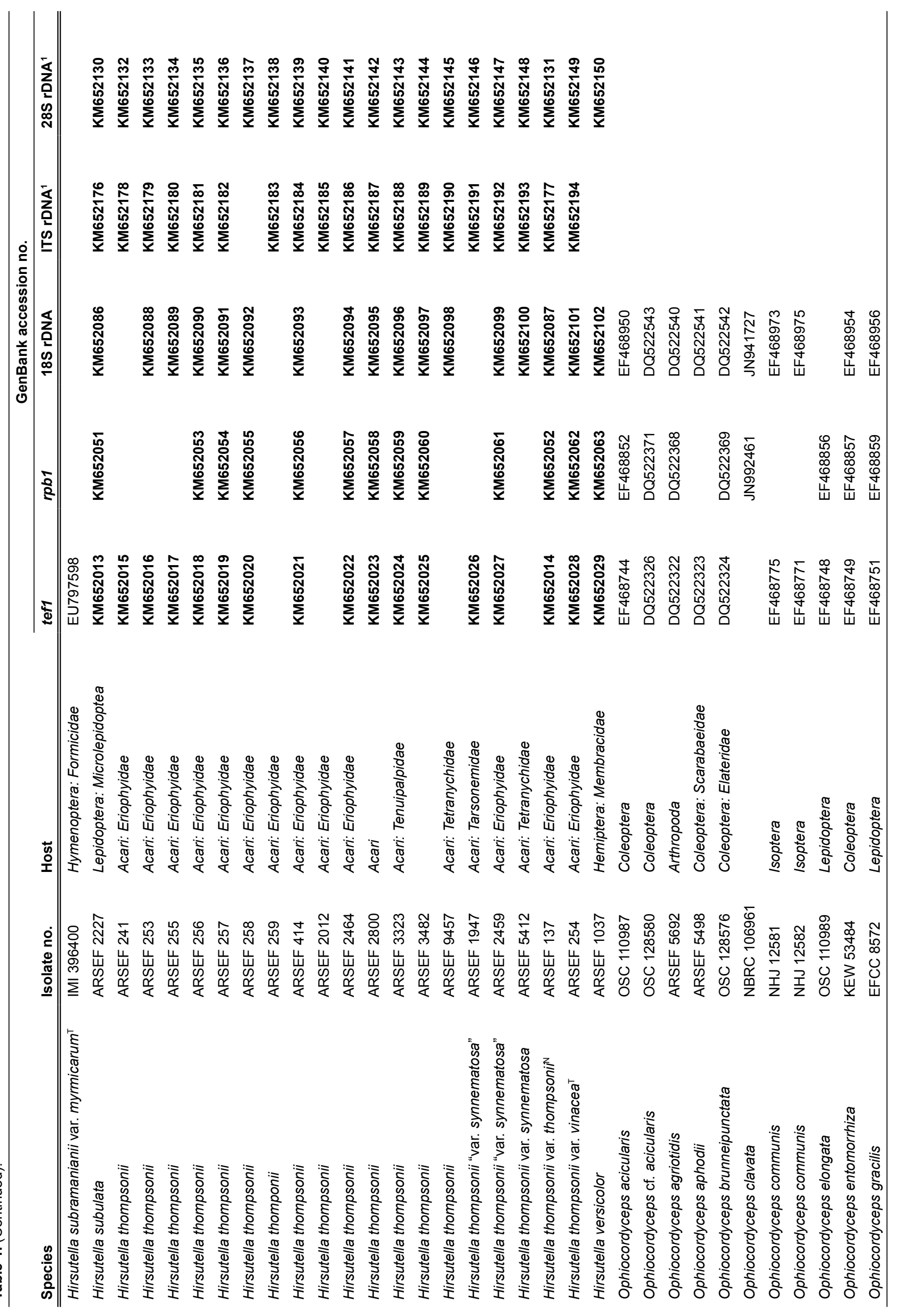




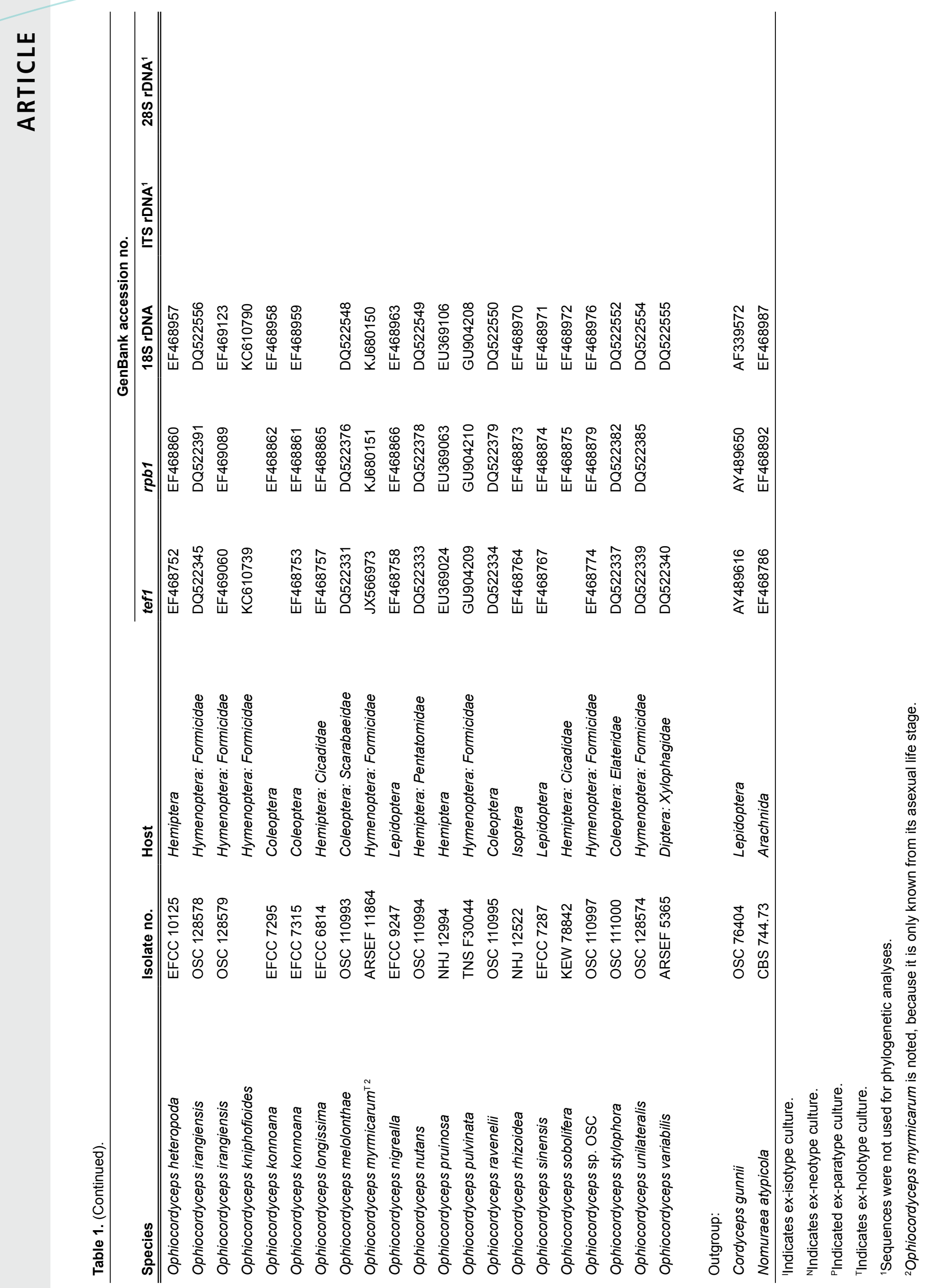




\section{DISCUSSION}

\section{Morphology}

Morphological characters of mitosporic reproductive structures (presence of synnemata; phialide appearance; conidia shape and condition) traditionally have been used as a basis for species description and hypothesized relatedness within Hirsutella. However, our data support Hodge's (1998) contention that the morphological features of Hirsutella are insufficient for determining interspecific relationships. Her subsequent phylogeny of the genus from nuclear ITS rDNA sequences ( $600 \mathrm{bp}$ each), including those obtained from eleven ARSEF isolates, seven of which are in this study, supported the monophyly of Hirsutella, so long as it includes three isolates of Harposporium, two of which would produce synanamorphs in culture studies. Based on our molecular phylogeny (Fig. 1), few trends in host taxa or morphological characters appear phylogenetically informative (Table 2). Some host based groupings can be inferred: the majority of the isolates from ant hosts cluster in one lineage, and species in the $H$. nodulosa clade primarily are associated with Lepidoptera hosts. However, other isolates originating from Lepidoptera are distributed within other clades, and isolates from Hemiptera hosts are distributed throughout all clades except one $(H$. nodulosa). Additional sampling of species from additional geographic regions or rare Hirsutella taxa, however, may expand these clades and make more relevant traits apparent.

\section{Hirsutella thompsonii clade}

Hirsutella thompsonii is the most widely studied of the Hirsutella species, being an important biocontrol agent for mite pests in agriculture. Isolates considered in this study, all originating from Acari hosts, form a clade including the morphologically similar species $H$. necatrix (Minter et al. 1983), another mite pathogen, and $H$. cryptosclerotium (Fernández-García et al. 1990), which originated from a different host taxon in Hemiptera. The monophyletic, core clade of $H$. thompsonii is composed of Hirsutella thompsonii var. thompsonii (ARSEF 137 - ex-neotype culture), most other $H$. thompsonii cultures, and $H$. thompsonii var. vinacea (ARSEF 254 - ex-holotype culture). Isolates ARSEF 1947 and 2459, which are included in the core clade, may form synnemata in culture (Humber et al. 2014), but they are not monophyletic with $H$. thompsonii var. synnematosa (ARSEF 5412), which is in a polytomy with $H$. necatrix and the core clade. Bayesian posterior probability support values deteriorate in the core clade because of differences in the placement of ARSEF 9457 by our analyses; Bayesian phylogenetic reconstruction removed ARSEF 9457 from the core clade and placed it in the polytomy alongside $H$. necatrix (ARSEF 5549 - ex-isotype culture) and $H$. thompsonii var. synnematosa. Additionally, ARSEF 258 and 2800 , also considered $H$. thompsonii, lie outside of the core clade, grouping with $H$. cryptosclerotium (ARSEF 4517 - ex-holotype culture). In our phylogeny isolates of Ophiocordyceps communis are sister to the taxa described above, most likely because of long-branch attraction instead of true phylogenetic relatedness; regardless, Sung et al. (2007) describe the asexual morph of O. communis to be transitionary between Hymenostilbe and Hirsutella.
Hodge (1998: 154 ) considered $H$. thompsonii to be ". . . the core of a group of morphologically related species including $H$. necatrix, $H$. gregis, $H$. cryptosclerotium, $H$. tydeicola, and $H$. sphaerospora, pathogens of mites or mealybugs." She examined the relationship of two $H$. thompsonii isolates, which are within our core clade, and $H$. cryptosclerotium, and she found $H$. cryptosclerotium to be sister to $H$. thompsonii with weak bootstrap support $(67 \%)$ in a nuclear ITS rDNA phylogeny. Hirsutella cryptosclerotium is distinguished by the production of pigmented sclerotia, but similar chlamydospore structures have been noted in other species, including $H$. thompsonii (Fernández-García et al. 1990). Hodge (1998) believed Hirsutella necatrix was more difficult to distinguish from $H$. thompsonii, except that the phialides were often formed in a verticillate arrangement and conidia were occasionally more ellipsoid.

Hodge (1998) posited that $H$. kirchneri and $H$. gregis (Minter et al. 1983), the latter of which we did not examine in this study, are variants from the same host and collection location. Similarly, Bałazy et al. (2008) produced phylogenies of nuclear ITS rDNA sequences that closely allied $H$. gregis and $H$. kirchneri isolates. A BLAST query of the nuclear ITS rDNA region generated from $H$. kirchneri (ARSEF 5551, GenBank KM652161) is $100 \%$ and $99 \%$ similar to the $H$. gregis and $H$. kirchneri isolates, respectively, as reported by Bałazy et al. (2008). The imperfect match of the two $H$. kirchneri sequences is explained by two ambiguous sites in the sequence determined by Bałazy et al. (2008) that are apparent in our ARSEF 5551 sequence. Hirsutella kirchneri and $H$. thompsonii are vastly disparate in our phylogeny, so the relationship of $H$. gregis to $H$. thompsonii within a morphologically defined 'core clade' as suggested by Hodge (1998) is not supported by our phylogeny.

Hirsutella tydeicola (Samson \& McCoy 1982) is known only from the type collections, but Hodge (1998) considered $H$. kirchneri and $H$. gregis similar to this isolate in appearance aside from slight variations in conidia shape, suggesting that $H$. tydeicola may be more closely related to $H$. kirchneri in our phylogeny and therefore disparate from $\mathrm{H}$. thompsonii. These morphological characters, however, may also prove misleading, and it could be that $H$. tydeicola resides in an unexpected location within the phylogeny; only a molecular characterization of this taxon will determine its phylogenetic position. Similarly, Hirsutella sphaerospora (Evans \& Samson 1982), which we did not examine, was theorized to be closely related to $H$. cryptosclerotium (Fernández-García et al. 1990, Hodge 1998), which also exhibits polyphialidic conidiogenous cells. The morphological association of $H$. sphaerospora to both $H$. cryptosclerotium and $H$. thompsonii may provide additional support for this species' placement within a 'core clade', but its true position is questionable.

\section{Hirsutella guyana clade}

The Hirsutella guyana clade is represented by three Hirsutella isolates characterized in our study. The ex-type culture of $H$. haptospora (Bałazy \& Wiśniewski 1986), which is considered in our phylogeny (ARSEF 2226), was isolated from mites in Formica nests from Poland. Hodge (1998) stated that this species was closely allied to $H$. rhossiliensis (Minter \& Brady 1980), but our phylogeny supports the placement of 


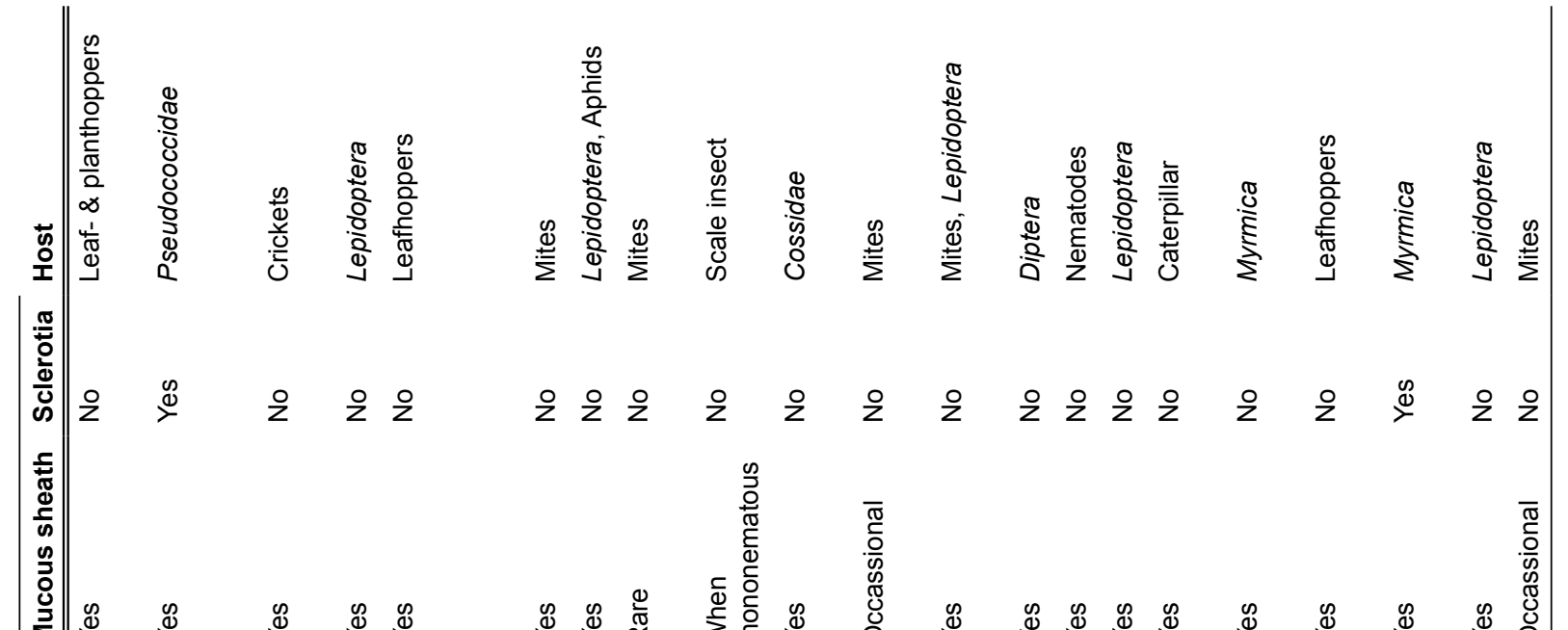

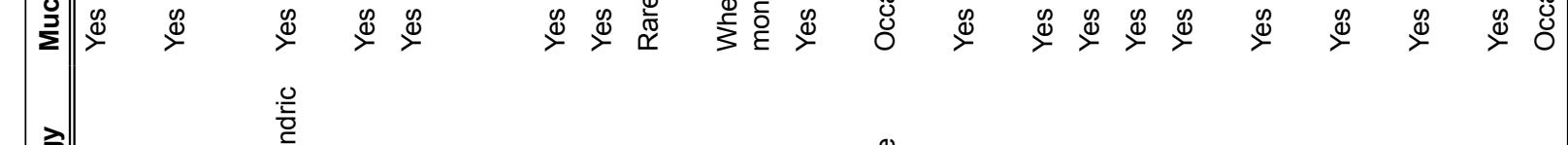

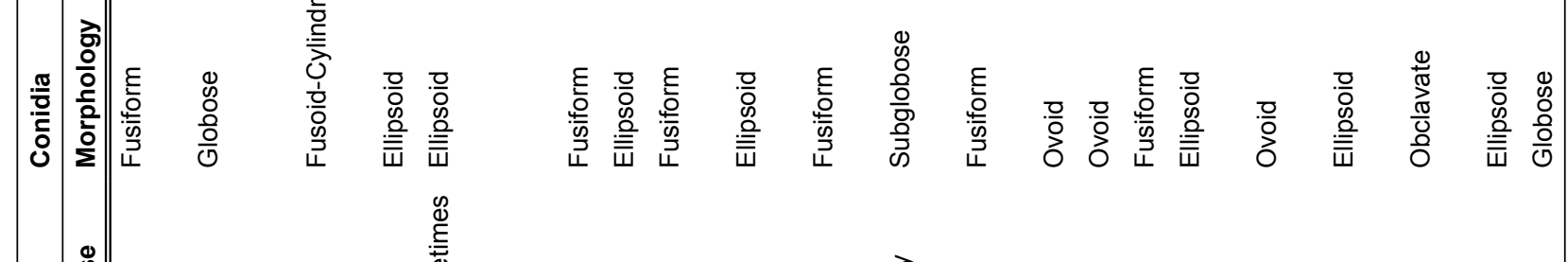
動

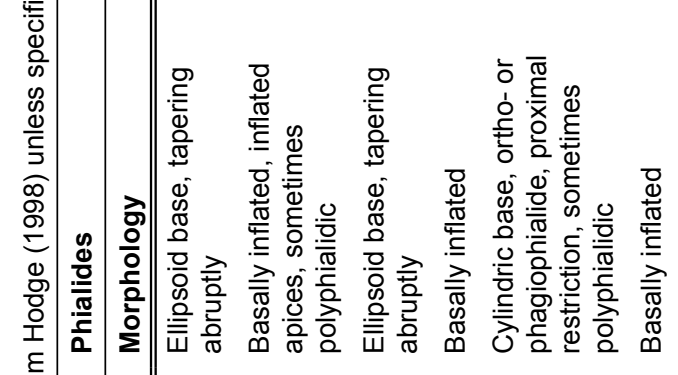

言

$\frac{\text { बे }}{\frac{\mathrm{e}}{\mathrm{w}}}$

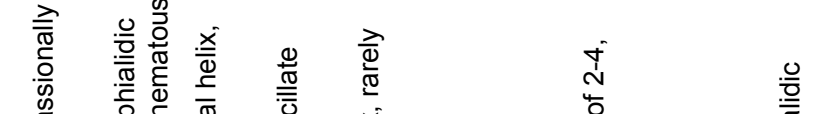




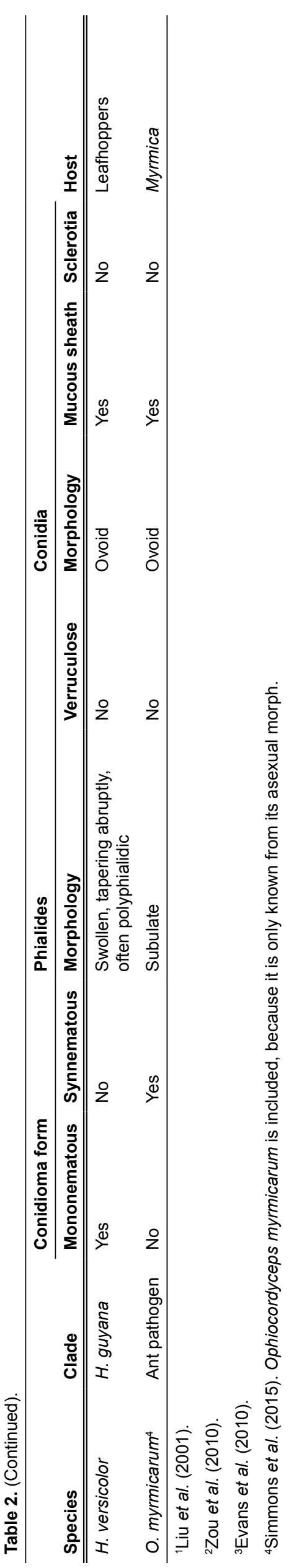

this species alongside O. pruinosa (Johnson et al. 2009) and a Brazilian isolate of H. guyana (Minter \& Brady 1980). Interestingly, H. cf. haptospora (ARSEF 2228), which was also identified by Bałazy from gall midges in Poland (Humber et al. 2014), is monophyletic with isolates of $H$. rhossiliensis. Hirsutella rhossiliensis is a broadrange nematode pathogen, but Hodge (1998) noted the morphological similarity of this species to $H$. haptospora. It is possible, therefore, that ARSEF 2228 represents a host-jumping $H$. rhossiliensis.

\section{Hirsutella nodulosa clade}

Four Hirsutella isolates clustered in the Hirsutella nodulosa clade. Hirsutella nodulosa (Petch 1926) and $H$. satumaensis (Aoki et al. 1957) are monophyletic with little variation in our phylogeny, which is similar to the findings of Hodge (1998) from a nuclear ITS rDNA dataset. Hirsutella nodulosa was described from a lepidopteran in Sri Lanka, and ARSEF 5473 is from a lepidopteran in Michigan. Minter \& Brady (1980) examined the type material and a culture from spider mites, and they suggested that the defining nodules described by Petch (1926) were conidia adhering by their mucilaginous sheath to hyphae, a condition that Hodge (1998) found to be a common occurrence in other Hirsutella species. Minter \& Brady (1980) also documented helical twisting at the apex of phialides and warts on phialides and hyphae, features not originally described by Petch (1926). Other species also may exhibit warted phialides (e.g. H. rhossiliensis, Minter \& Brady 1980) though not to the degree seen in H. nodulosa. Hirsutella brownorum (Minter \& Brady 1980) and H. liboensis (Zou et al. 2010), the latter of which groups with $H$. nodulosa in our phylogeny, also possess phialides with apical helical twists. The determination of the phylogenetic position of $H$. brownorum, and additional taxa with apical helical twists (Zou et al. 2010), however, would be necessary before concluding that this trait evolved only once.

Hirsutella satumaensis was described from case-making clothes moths and silkworms, and ARSEF 996 is derived from one of Aoki's cultures of this species (Aoki et al. 1957). Hodge (1998), however, saw no evidence for this isolate to be defined as $H$. satumaensis, reported no other isolates or type material, and believed ARSEF 996 to be $H$. nodulosa. We support Hodge's hypothesis, and the phylogenetic position of $H$. satumaensis will remain unknown until a neotype fitting the description can be studied.

Ciancio et al. (2013) described $H$. tunicata from Peruvian mites as being similar in appearance to $H$. nodulosa, but $H$. tunicata lacks the twisted apex of the phialides and possesses solitary conidia. In phylogenies from nuclear ITS rDNA and $\beta$-tubulin gene datasets produced by Ciancio et al. (2013), $H$. tunicata allied with an isolate of $H$. nodulosa from strawberry mites in Poland, but support values for this grouping were low, suggesting that morphological similarities between these species are not necessarily indicative of true relatedness. However, a BLAST query in GenBank of the nuclear ITS rDNA sequence obtained by Ciancio et al. (2013) from H. tunicata is best matched at $93 \%$ identity with the sequence we generated from $H$. subulata (Petch 1932) ARSEF 2227 (GenBank KM652176), which is also within the $H$. nodulosa clade. The relationship hypothesized by Ciancio et al. (2013), therefore, has yet to be disproven.

Our phylogeny also supports previous analyses (Simmons et al. 2015) in which comparable isolates of $O$. acicularis (Petch 1933) are not monophyletic, with representatives in both the $H$. nodulosa and $H$. sinensis clades. Ophiocordyceps cf. acicularis (OSC 128580), however, appears synonymous with $H$. liboensis in our phylogeny. Zou et al. (2010) believed $H$. liboensis could be the asexual morph of Ophiocordyceps cochlidiicola (Sung et al. 2007), the taxon for which H. liboensis was the closest relative in the nuclear ITS rDNA phylogeny produced by Zou et al. (2010), next only to an isolate of $H$. nodulosa. Additionally, Sung et al. (2007) placed Cordyceps cf. acicularis (as O. cf. acicularis) sister to an isolate of O. cochlidiicola in five-gene phylogenies with $100 \%$ support. The alliance of $H$. liboensis and $O$. cochlidiicola, in addition to their relatedness to $H$. nodulosa, seem highly likely, but we agree with Zou et al. (2010) that further study is necessary to confirm this relationship.

\section{Hirsutella sinensis clade}

The Hirsutella sinensis clade includes isolates originating from a variety of taxa including nematodes, mites, and both hemi- (Hemiptera) and holometabolist 
(Coleoptera, Lepidoptera) insect hosts. Our data supports the evidence of Liu et al. (2001) that $H$. sinensis (Liu et al. 1989 ) is the anamorph of Cordyceps sinensis (Sung et al. 2007). Liu et al. (2001, as C. sinensis) used nuclear ITS rDNA sequences and morphological examination of ascospore microcyclic conidia of $C$. sinensis (Saccardo 1878) to argue that $H$. sinensis was the genuine asexual morph of $C$. sinensis, for which several names based on asexual morphs had been suggested.

Hirsutella strigosa (Petch 1939) forms a monophyletic group with $H$. repens (nom. inval.; Humber et al. 2014), for which no morphological data is published, and $H$. kirchneri, with which it shares some morphological features. Petch (1939) found $H$. strigosa with $H$. versicolor, and our phylogeny clearly differentiates these taxa. Hodge (1998) notes a distinguishing feature of this species is its exceptionally long and verruculose phialides, similar to $H$. nodulosa, but not apically twisted as in that species. Furthermore, Hodge noted that $H$. illustris and $H$. rhossiliensis, also within our $H$. sinensis clade, similarly share large phialides. $H$. kirchneri and $H$. lecaniicola (Petch 1933), however, do not share this characteristic.

\section{Hirsutella citriformis clade}

Hirsutella citriformis (Speare 1920) is a widely distributed species infecting the Asian citrus psyllid Diaphorina citri (e.g. Subandiyah et al. 2000, Hall et al. 2012, Pérez-González et al. 2015) and is represented in our phylogeny by isolates collected in the Republic of Indonesia, Republic of the Philippines, and the USA. This species is monophyletic, indicating reliable morphological identification by different investigators, but Pérez-González et al. (2015) argued that morphologies of phialides and conidia of isolates vary from those of the original description and warn that new isolates could show increasing variability in these structures.

Other isolates in this clade originate from a diversity of insect taxa. Hirsutella fusiformis (Speare 1920) is poorly known from the type description and illustrations; Hodge (1998) examined the putative isolate of $H$. fusiformis we investigated in this study and found that it would not sporulate on tested media. In our phylogeny, $H$. fusiformis was associated with $H$. radiata (Petch 1935). Hodge (1998) postulated that $H$. guignardii (Samson et al. 1984) could be a synonym of $H$. radiata, based on the similarity of synnemata branching, though more delicate in the latter species. It is possible, based on the short branch lengths in our phylogeny comparable to other taxonomically identical taxa, that this putative $H$. fusiformis is instead one of these species but has degenerated in storage. Additionally in this clade, Simmons et al. (2015) previously confirmed the relationship of Hirsutella gigantea (Petch 1937) and Ophiocordyceps elongata (Sung et al. 2007) based on tef1 data, and our wider phylogeny including an rpb1 sequence obtained from this isolate provides further support for the association.

\section{Hirsutella ant pathogen clade}

Our phylogeny places all Hirsutella isolates from Formicidae in a monophyletic clade. This clade, however, excludes isolates of Ophiocordyceps irangiensis, which, while also derived from Formicidae hosts, produce a typical Hymenostilbe asexual morph (Sung et al. 2007). Simmons et al. (2015) noted similar phylogenetic relationships of these taxa in their $\mathrm{ML}$ analysis of tef1, rpb1, and 18S rDNA, but a Bayesian phylogeny of those same loci grouped $O$. irangiensis with isolates of $O$. communis, which produces a transitionary HirsutellalHymenostilbe asexual morph (Sung et al. 2007).

\section{An ongoing investigation}

Hodge (1998) produced the only monographic work on Hirsutella that incorporated genetic sequences for an initial phylogenetic understanding of the genus. Our examination of ARSEF Hirsutella isolates from the USA sought to increase the sampling of these fungi in molecular databases, but a monographic revision of this genus would be incomplete if it did not consider the entirety of Ophiocordyceps, with which Hirsutella species are being synonymized. Indeed, more rigorous morphological examinations of the Hirsutella isolates in our molecular phylogeny are necessary before taxonomic revisions (combinations, typifications, etc.) can take place, but our molecular phylogeny provides an evolutionary context for morphological features and physiology and a measure of species' similarity that will facilitate the next steps in this process. Additionally, phylogenetic investigations into international or rare Hirsutella species and isolates, which we were unable to examine in this study, will likely lead to a deeper understanding of the evolution, taxonomy, and physiology of these fungi.

\section{ACKNOWLEDGEMENTS}

We thank Richard A Humber for access to the ARSEF Culture Collection and his expert advice; Patty Singer and Dave Cox of the DNA Sequencing Facility at the University of Maine for their services. We also thank Joyce $E$ Longcore for participation in taxonomic and phylogenetic discussions, and Jerry $\mathrm{R}$ Longcore for critical consultation in preparing the manuscript. This project was supported by the Agricultural and Food Research Initiative Competitive Grant No. 2013-67012-21115 from the USDA National Institute of Food and Agriculture, and the Maine Agriculture and Forestry Experiment Station at the University of Maine. This is MAFES Publication No. 3438.

\section{REFERENCES}

Aoki K, Nakasato Y, Fujimoto I, Suzuki H (1957) Studies on the new fungous parasites of silkworms, Bombyx mori L. IV. Hirsutella satumaensis Aoki sp. nov. Bulletin of the Sericultural Experiment Station 14: 471-486.

Bałazy S, Wiśniewski J (1986) Two new species of Hirsutella infecting mites in Poland. Transactions of the British Mycological Society 86: 629-635.

Bałazy S, Wrzosek M, Sosnowska D, Tkaczuk C, Muszewska A (2008) Laboratory trials to infect insects and nematodes by some acaropathogenic Hirsutella strains (Mycota: Clavicipitaceous anamorphs). Journal of Invertebrate Pathology 97: 103-113.

Carbone I, Kohn LM (1999) A method of designing primer sets for speciation studies in filamentous ascomycetes. Mycologia 91: 553-556.

Castlebury LA, Rossman AY, Sung G-H, Hyten AS, Spatafora JW (2004) Multigene phylogeny reveals new lineage for Stachybotrys 
chartarum, the indoor air fungus. Mycological Research 108: 864-872.

Chaverri P, Samuels GJ, Hodge KT (2005) The genus Podocrella and its nematode-killing anamorph Harposporium. Mycologia 97: 433-443.

Ciancio A, Colagiero M, Rosso LC, Murga Gutierrez SN, Grasso G (2013) Phylogeny and morphology of Hirsutella tunicata sp. nov. (Ophiocordycipitaceae), a novel mite parasite from Peru. Mycoscience 54: 378-386.

Evans HC, Groden E, Bischoff JF (2010) New fungal pathogens of the red ant, Myrmica rubra, from the UK and implications for ant invasions in the USA. Fungal Biology 114: 451-466.

Evans HC, Samson RA (1982) Entomogenous fungi from the Galápagos Islands. Canadian Journal of Botany 60: 23252333.

Fernández-García E, Evans HC, Samson RA (1990) Hirsutella cryptosclerotium sp. nov., an entomopathogen of the mealybug pest, Rastrococcus invadens, in West Africa. Mycological Research 94: 1111-1117.

Gardes M, Bruns TD (1993) ITS primers with enhanced specificity for basidiomycetes: application to the identification of mycorrhizae and rusts. Molecular Ecology 2: 113-118.

Guindon S, Gascuel O (2003) A simple, fast, and accurate algorithm to estimate large phylogenies by maximum likelihood. Systematic Biology 52: 696-704.

Hall DG, Hentz MG, Meyer JM, Kriss AB, Gottwald TR, Boucias DG (2012) Observations on the entomopathogenic fungus Hirsutella citriformis attacking adult Diaphorina citri (Hemiptera: Psyllidae) in a managed citrus grove. BioControl 57: 663-675.

Hodge KT (1998) Revisionary studies in Hirsutella (anamorphic Hypocreales: Clavicipitaceae). PhD dissertation, Cornell University, USA.

Humber RA, Hansen K, Wheeler MM (2014) ARSEF USDAARS Collection of Entomopathogenic Fungal Cultures: Catalog of species. http://www.ars.usda.gov/Main/docs. $\mathrm{htm}$ ?docid=12125\&page $=2$

Johnson D, Sung G-H, Hywel-Jones NL, Luangsa-Ard JJ, Bischoff JF, Kepler RM, Spatafora JW (2009) Systematics and evolution of the genus Torrubiella (Hypocreales, Ascomycota). Mycological Research 113: 279-289.

Kepler RM, Ban S, Nakagiri A, Bischoff JF, Hywel-Jones NL, Owensby CA, Spatafora JW (2013) The phylogenetic placement of hypocrealean insect pathogens in the genus Polycephalomyces: an application of One Fungus One Name. Fungal Biology 117: 611-622.

Lanfear R, Calcott B, Ho SYW, Guindon S (2012) PartitionFinder: combined selection of partitioning schemes and substitution models for phylogenetic analyses. Molecular Biology and Evolution 29: 1695-1701.

Liu X-J, Guo Y-L, Yu Y-X, Zeng W (1989) Isolation and identification of the anamorphic stage of Cordyceps sinesis (Berk.) Sacc. Acta Mycologica Sinica 8: 35-40.

Liu Z, Yao Y, Liang ZQ, Liu A, Pegler DN, Chase MW (2001) Molecular evidence for the anamorph - teleomorph connection in Cordyceps sinensis. Mycological Research 105: 827-832.

McNeill J, Barrie FF, Buck WR, Demoulin V, Greuter W, et al. (eds) (2012) International Code of Nomenclature for algae, fungi, and plants (Melbourne Code). [Regnum vegetabile no. 154.] Königstein: Koeltz Scientific Books.

Minter DW, Brady BL (1980) Mononematous species of Hirsutella. Transactions of the British Mycological Society 74: 271-282.
Minter DW, Brady BL, Hall RA (1983) Five hyphomycetes isolated from eriophyid mites. Transactions of the British Mycological Society 81: 455-471.

Patouillard NT (1892) Une Clavariée entomogène. Revue Mycologique 14: 67-70.

Pérez-González O, Rodríguez-Villarreal RA, López-Arroyo Jl, Maldonado-Blanco MG, Rodríguez-Guerra R (2015) Mexican strains of Hirsutella isolated from Diaphorina citri (Hemiptera: Liviidae): morphologic and molecular characterization. Florida Entomologist 98: 290-297.

Petch T (1926) Entomogenous fungi. Additions and corrections, II. Transactions of the British Mycological Society 11: 258-266.

Petch T (1932) British species of Hirsutella. The Naturalist (London) 1932: 45-49.

Petch T (1933) Notes on entomogenous fungi. Transactions of the British Mycological Society 18: 48-75.

Petch T (1935) Notes on entomogenous fungi. Transactions of the British Mycological Society 19: 161-194.

Petch T (1937) Notes on entomogenous fungi. Transactions of the British Mycological Society 21: 34-67.

Petch T (1939) Notes on entomogenous fungi. Transactions of the British Mycological Society 23: 127-148.

Posada D (2008) jModeltest: phylogenetic model averaging. Molecular Biology and Evolution 25: 1253-1256.

Quandt CA, Kepler RM, Gams W, Araújo JPM, Ban S, et al. (2014) Phylogenetic-based nomenclatural proposals for Ophiocordycipitaceae (Hypocreales) with new combinations in Tolypocladium. IMA Fungus 5: 121-134.

Rehner SA, Buckley E (2005) A Beauveria phylogeny inferred from nuclear ITS and EF1- $\alpha$ sequences: evidence for cryptic diversification and links to Cordyceps teleomorphs. Mycologia 97: 84-98.

Rehner SA, Samuels GJ (1994) Taxonomy and phylogeny of Gliocladium analysed from nuclear large subunit ribosomal DNA sequences. Mycological Research 98: 625-634.

Ronquist F, Huelsenbeck JP (2003) MrBayes 3: Bayesian phylogenetic inference under mixed models. Bioinformatics 19: 1572-1574.

Saccardo PA (1878) Enumeratio pyrenomycetum hypocreaceorum hucusque congnitorum systemate carpologico dispositorum. Michelia 1: 277-325.

Samson RA, McCoy CW (1982) A new fungal pathogen of the scavenger mite, Tydeus gloveri. Journal of Invertebrate Pathology 40: 216-220.

Samson RA, Rombach MC, Seifert KA (1984) Hirsutella guignardiiand Stilbella kervillei, two troglobiotic entomogenous hyphomycetes. Persoonia 12: 123-134.

Seifert KA, Boulay H (2004) Hirsutella uncinata, a new hyphomycete from Australia. Mycologia 96: 929-934.

Simmons DR (2011) Phylogeny of Powellomycetaceae fam. nov. and description of Geranomyces variabilis gen. et comb. nov. Mycologia 103: 1411-1420.

Simmons DR, Lund J, Levitsky T, Groden E (2015) Ophiocordyceps myrmicarum, a new species infecting invasive Myrmica rubra in Maine. Journal of Invertebrate Pathology 125: 23-30.

Speare AT (1920) On certain entomogenous fungi. Mycologia 12: 62-76.

Subandiyah S, Nikon N, Sato H, Wagiman F, Tsuyumu S, Fakatsu $T$ (2000) Isolation and characterization of 2 entomopathogenic fungi attacking Diaphorina citri (Homoptera, Psilloidea) in Indonesia. Mycoscience 41: 509-513. 
Sukumaran J, Holder MT (2010) DendroPy: a python library for phylogenetic computing. Bioinformatics 26: 1569-1571.

Sung G-H, Hywel-Jones NL, Sung J-M, Luangsa-ard JJ, Shrestha B, Spatafora JW (2007) Phylogenetic classification of Cordyceps and the clavicipitaceous fungi. Studies in Mycology 57: 5-59.

Vilgalys R, Hester M (1990) Rapid genetic identification and mapping of enzymatically amplified ribosomal DNA from several Cryptococcus species. Journal of Bacteriology 172: 4238-4246.

White TJ, Bruns TD, Lee SB, Taylor JW (1990)Amplification and direct sequencing of fungal ribosomal RNA genes for phylogenetics. In: PCR Protocols: a guide to methods and applications (Innis MA, Gelfand DH, Sninsky JJ, White TJ, eds): 315-322. San Diego: Academic Press.
Zou X, Liu A, Liang Z, Han Y, Yang M (2010) Hirsutella liboensis, a new entopathogenic species affecting Cossidae (Lepidoptera) in China. Mycotaxon 111: 39-44.

Zwickl DJ (2006) Genetic algorithm approaches for the phylogenetic analysis of large biological sequence datasets under the maximum likelihood criterion. PhD dissertation, The University of Texas at Austin. 